\title{
Effect of initial retinal thickness on outcome of intravitreal bevacizumab therapy for diabetic macular edema
}

This article was published in the following Dove Press journal:

Clinical Ophthalmology

28 April 2014

Number of times this article has been viewed

\author{
Bushra Mushtaq ${ }^{1, *}$ \\ Niall J Crosby $1, *$ \\ Antonios T Dimopoulos' \\ Peck Lin Lip' \\ Panagiota Stavrou' \\ Samer El-Sherbiny' \\ Yit Yang ${ }^{2}$ \\ 'Birmingham and Midland Eye \\ Centre, City and Sandwell National \\ Health Service Trust, Birmingham, \\ West Midlands, UK; ${ }^{2}$ Life and \\ Health Sciences, Aston University, \\ Birmingham, West Midlands, UK \\ *These authors contributed equally \\ to this work
}

Purpose: To investigate whether eyes with diabetic macular edema (DME) and central retinal thickness $(\mathrm{CRT})>400 \mu \mathrm{m}$ had better visual and anatomical outcomes compared to eyes with a CRT $<400 \mu \mathrm{m}$ when treated with intravitreal bevacizumab in a real-world setting.

Patients and methods: Patients undergoing intravitreal bevacizumab therapy for DME were identified from the departmental database of a tertiary referral unit. Following the initial injection, a retreatment was performed for any persistent macular edema, unless there had been no previous response to repeated doses. Recorded parameters included visual acuity, CRT on optical coherence tomography (spectral domain optical coherence tomography [SD-OCT]), and SD-OCT characteristics. Comparisons were made between data at baseline and 12 months after the first injection, and differences were tested for statistical significance using the Student's $t$-test.

Results: In all, 175 eyes of 142 patients were analyzed. Patients in group 2 (CRT $>400 \mu \mathrm{m})$ had significantly more injections than group $1(\mathrm{CRT}<400 \mu \mathrm{m})(4.0$ versus $3.3 ; P=0.003)$. Both groups had similar numbers of eyes with preexisting epiretinal membrane and/or vitreomacular traction at baseline. The reduction in CRT was significantly greater in group 2 when compared to group $1(P<0.0001)$. In terms of visual gain between baseline and month 12 , each gained significantly by a mean of 0.12 logarithm of the minimum angle of resolution units $(P=0.0001)$, but there was no difference between groups 1 and $2(P=0.99)$.

Conclusion: These results do not support a $400 \mu \mathrm{m}$ baseline CRT cut-off for treating DME with bevacizumab, in contrast to published data on ranibizumab. Our results also indicate that patients with a thicker CRT require more bevacizumab injections, making treatment less costeffective for these patients. Our results could be used by practitioners to support the use of bevacizumab in DME without applying a CRT cut-off.

Keywords: anti-VEGF therapy, central retinal thickness, ranibizumab, intravitreal injection, optical coherence tomography

\section{Introduction}

Diabetic macular edema (DME) is the leading cause of vision loss in patients with diabetes. ${ }^{1}$ Several studies have reported raised levels of vascular endothelial growth factor (VEGF) in the aqueous humor of eyes with DME when compared with healthy eyes. $^{2-4}$ Anti-VEGF therapy with pegaptanib sodium, ranibizumab, bevacizumab, and aflibercept has been shown to have beneficial effects in terms of a reduction in edema and central retinal thickening as well as in terms of vision gain or stability. ${ }^{5-11}$

On February 27, 2013, the National Institute of Clinical Excellence in the UK recommended the use of ranibizumab for DME but only in the subgroup of patients with central retinal thickness (CRT) of $>400 \mu \mathrm{m} .{ }^{12}$ This decision was based partly on the evidence from recent randomized clinical trials, which showed that eyes 
with $\mathrm{CRT}>400 \mu \mathrm{m}$ at baseline had more improvement in terms of visual acuity (VA) and CRT than those with CRT of $<400 \mu \mathrm{m}^{.10,13}$

With regards to the bevacizumab therapy for DME, however, numerous studies have reported varying visual outcomes. ${ }^{8,14-16}$ Several studies have found that the pattern of edema classified on spectral domain optical coherence tomography (SD-OCT) has a significant influence on treatment response and visual outcome. ${ }^{15-18}$

However, only one study to date has shown a greater treatment response to bevacizumab in eyes with a higher baseline CRT $>350 \mu \mathrm{m} .{ }^{19}$ Given that the lower cost of small aliquots of bevacizumab from a compounding pharmacy has made bevacizumab a popular choice for intravitreal antiVEGF therapy in many countries, ${ }^{20}$ we therefore wanted to investigate whether eyes with DME and CRT $>400 \mu \mathrm{m}$ had better visual and anatomical outcomes when treated with bevacizumab in a real-world setting. This type of evidence may not only support the rationale of applying a CRT cut-off in treating DME with anti-VEGF therapy, but it may also be useful to clinicians in health care settings where patients have to opt for either ranibizumab or bevacizumab for personal economic reasons. In this study, we report visual and anatomical outcomes of eyes with DME treated with intravitreal bevacizumab on a pro re nata basis at a single tertiary center, and we also compare visual outcomes at 1 year.

\section{Materials and methods}

The setting for this study was an intravitreal bevacizumab treatment service in a tertiary referral unit, provided by four specialists (PLL, SE, PS, BM) for patients with DME. Patients were referred from clinics within the Birmingham and Midland Eye Centre and from surrounding hospitals by their local ophthalmologists. Only the patients with center involving diffuse cystoid DME, or those that were unlikely to benefit from laser, were accepted into the treatment program.

Within the treatment program, eyes with DME were managed according to a standard treatment algorithm: 1) all patients had a baseline VA test, clinical examination, and SDOCT scan (Topcon 3D OCT 1000; TOPCON Corporation, Tokyo, Japan); 2) after written informed consent, patients received intravitreal bevacizumab $(1.25 \mathrm{mg}$ in $0.05 \mathrm{~mL})$ under standard aseptic conditions (bevacizumab supplies were from Moorfields Eye Hospital's compounding pharmacy); 3) following the first baseline treatment, patients attended one to three monthly monitoring visits for VA, biomicroscopy, and SD-OCT scan; 4) after the first injection, retreatment was performed for any persistent macular edema on SD-OCT unless there had been no additional response observed after repeated dosing in previous visits; and 5) eyes that had minimal or no response to intravitreal bevacizumab in terms of SD-OCT appearance were considered by the treating physician at any monitoring visit for intravitreal triamcinolone if this had not been tried prior to bevacizumab, as other intravitreal agents - such as ranibizumab, fluocinolone, and aflibercept - were not available at the time of the study.

From this treatment program, consecutive patients undergoing intravitreal bevacizumab therapy for DME in at least one eye were identified from the departmental database. To obtain an adequate sample size of patients with up to 1 year of follow-up, patients who had their first injection from March 1, 2009-January 25, 2012, were included. Data capture was performed in March-April 2013 by means of retrospective chart review to obtain biographical, VA, and other data on injections and follow-up visits. The baseline was defined as the visit when the first intravitreal bevacizumab was administered. The final visit was defined as the visit that was either at 12 months from the baseline or the closest visit beyond the 12-month time point. Patients who had been discharged earlier than 12 months back to their local ophthalmologists in the surrounding hospital were excluded. Data on the number of intravitreal injections and the VA and the CRT at baseline and at the final 1-year time point were extracted from the charts and the SD-OCT database. In addition, baseline SD-OCT scans were retrospectively regraded to determine the presence of epiretinal membrane and vitreomacular traction, which are features known to be associated with a poor response.

To evaluate the differential treatment response of severe and less severe DME, patients were divided into group 1 $(<400 \mu \mathrm{m})$ and group $2(>400 \mu \mathrm{m})$. Comparisons were made of CRT and VA between the groups and also within the groups (final versus baseline). Two sample, two-sided, unpaired Student's $t$-tests were used to make comparisons between the two groups; one-sided, paired Student's $t$-tests were used to compare the change in CRT and the VA within each group. Data analysis was performed using the Statistical Package for Social Sciences software (SPSS Inc., Chicago, IL, USA). $P$-values of $<0.05$ were considered statistically significant.

\section{Results}

Data on 195 eyes of 159 patients were collected. Also, 20 eyes of 17 patients were excluded due to the following reasons: insufficient follow-up (eight eyes, six patients); lack of final $\mathrm{SD}-\mathrm{OCT}$ (four eyes, four patients); delay in 12-month review 
date by more than 2 months (three eyes, two patients); coexisting retinal vein occlusion (two eyes, two patients); and missing data on acuity or treatment dates (three eyes, three patients). A total of 175 eyes of 142 patients were included in analysis. All eyes were confirmed on retrospective SD-OCT regrading to have a cystoid pattern of DME with cysts in either inner or outer retinal layers.

Of these, 81 eyes of 66 patients had CRT $<400 \mu \mathrm{m}$ (group 1) and 94 eyes of 76 patients had CRT $>400 \mu \mathrm{m}$ (group 2). The baseline characteristics of these two groups of eyes and their exposure to treatment over the 12-month period are shown in Table 1. Both groups had similar numbers of eyes with preexisting epiretinal membrane and/or vitreomacular traction visible on SD-OCT at the baseline. Although very few patients required intravitreal triamcinolone during the study period, more patients in group 2 required it. Four patients in group 1 received intravitreal triamcinolone compared to eight in group 2 during the study period. Group 2 patients had significantly more injections than group 1 patients ( 4.0 versus $3.3 ; P=0.003$ ). The results for CRT are shown in Table 2. Both groups showed a significant reduction in CRT between the baseline and month 12 . The amount of reduction in CRT was significantly greater in group 2 when compared to group $1(P<0.0001)$.

In terms of VA, both groups had comparable visual acuities at the baseline and gained by a mean of 0.12 logarithm of the minimum angle of resolution (logMAR) units (equivalent to six letters) between baseline and month 12 . The difference between groups 1 and 2 in terms of visual gain was not statistically significant $(P=0.99)$. Within each group, however, there was an improvement in the VA from the baseline to the

Table I Distribution of patients, eyes, and treatment in groups I and 2

\begin{tabular}{|c|c|c|}
\hline Parameter & 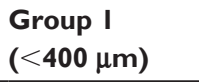 & $\begin{array}{l}\text { Group } 2 \\
(>400 \mu \mathrm{m})\end{array}$ \\
\hline Number of patients ( $\%$ female) & $66(46 \%)$ & $76(33 \%)$ \\
\hline Mean age in years (range) & $63(19-87)$ & $63(27-87)$ \\
\hline $\begin{array}{l}\text { Mean age - group comparison } \\
\text { (unpaired Student's } t \text {-test) }\end{array}$ & $P=0.34$ & \\
\hline $\begin{array}{l}\text { Number of bilaterally treated } \\
\text { patients }\end{array}$ & 15 & 18 \\
\hline Total number of eyes & 81 eyes & 94 eyes \\
\hline Eyes with ERM or VMT at baseline & $16 / 83(19.3 \%)$ & $23 / 94(24.5 \%)$ \\
\hline $\begin{array}{l}\text { Mean number of injections: } \\
\text { (mode, range, total) }\end{array}$ & $3.3(3,1-8,272)$ & $4.0(3,1-9,375)$ \\
\hline $\begin{array}{l}\text { Number of injections - group } \\
\text { comparison (unpaired Student's t-test) }\end{array}$ & $P=0.003$ & \\
\hline
\end{tabular}

Abbreviations: ERM, epiretinal membrane; VMT, vitreomacular traction.
Table 2 CRT at baseline and 12 months in groups I and 2

\begin{tabular}{|c|c|c|}
\hline & 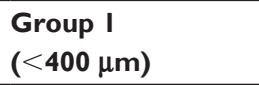 & $\begin{array}{l}\text { Group } 2 \\
(>400 \mu \mathrm{m})\end{array}$ \\
\hline $\begin{array}{l}\text { Mean baseline CRT in } \mu \mathrm{m} \\
(\mathrm{SD} \text {, range) }\end{array}$ & $326(43.8,232-395)$ & $539(106.3,402-846)$ \\
\hline $\begin{array}{l}\text { Group difference } \\
\text { (unpaired Student's } t \text {-test) }\end{array}$ & $213 ; P<0.0001 ;(P=6.2$ & $\left.2 \times 10^{-39}\right)$ \\
\hline $\begin{array}{l}\text { Mean final CRT in } \mu \mathrm{m} \\
\text { (SD, range) }\end{array}$ & $293(69.3,134-475)$ & $393(128.5,166-776)$ \\
\hline $\begin{array}{l}\text { Group difference } \\
\text { (unpaired Student's t-test) }\end{array}$ & $100, P<0.0001$ & \\
\hline $\begin{array}{l}\text { Mean reduction in } \\
\mathrm{CRT} \text { in } \mu \mathrm{m} \text { (SD, range) } \\
\text { (minus denotes worsening) } \\
\text { (paired Student's } t \text {-test) }\end{array}$ & $\begin{array}{l}33.6,(75.6 ;-185 \\
\text { to }+220) P=0.0001\end{array}$ & $\begin{array}{l}\text { I46, }(|32.8 ;-2| \text { I } \\
\text { to }+570) P<0.000 \text { I }\end{array}$ \\
\hline $\begin{array}{l}\text { Mean between group } \\
\text { reduction in CRT in } \mu \mathrm{m} \\
\text { (unpaired Student's } t \text {-test) }\end{array}$ & $112.4 ; P<0.000 \mid$ & \\
\hline
\end{tabular}

final of $0.12 \log$ MAR units. This was statistically significant $(P=0.0001)$. The VA results are shown in Table 3 .

\section{Discussion}

The results from this study demonstrated the treatment response that could be achieved with intravitreal bevacizumab, as both groups of eyes showed a significant improvement in CRT measurements, with the greatest anatomical response in the group with CRT $>400 \mu \mathrm{m}$. This finding of a differential anatomical response depending on baseline CRT is in keeping with one study using bevacizumab by Soheilian et al and also in studies using ranibizumab. ${ }^{13,19,21}$ Evidence for a differential response in terms of visual gain is less clear. In this study,

Table $3 \mathrm{VA}$ at baseline and at 12 months in groups I and 2

\begin{tabular}{|c|c|c|}
\hline & 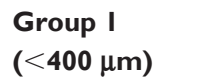 & $\begin{array}{l}\text { Group } 2 \\
(>400 \mu \mathrm{m})\end{array}$ \\
\hline $\begin{array}{l}\text { Mean baseline acuity - logMAR } \\
\text { units (SD, range) }\end{array}$ & $\begin{array}{l}0.58(\approx 20 / 80) \\
(0.31,0.18-1.50)\end{array}$ & $\begin{array}{l}0.67(\approx 20 / 100) \\
(0.35,0.18-1.78)\end{array}$ \\
\hline $\begin{array}{l}\text { Mean baseline acuity - difference } \\
\text { between groups (unpaired } \\
\text { Student's } t \text {-test) }\end{array}$ & $0.09, P=0.06$ & \\
\hline $\begin{array}{l}\text { Mean final acuity in logMAR } \\
\text { units (SD, range) }\end{array}$ & $\begin{array}{l}0.45(\approx 20 / 63) \\
(0.29,0.00-1.3)\end{array}$ & $\begin{array}{l}0.55(\approx 20 / 80) \\
(0.33,0.00-1.78)\end{array}$ \\
\hline $\begin{array}{l}\text { Mean final acuity - difference } \\
\text { between groups (unpaired } \\
\text { Student's } t \text {-test) }\end{array}$ & $0.10, P=0.049$ & \\
\hline $\begin{array}{l}\text { Mean gain in VA within group } \\
\text { (SD, range) (minus denotes visual }\end{array}$ & $\begin{array}{l}+0.12(0.28 \\
-0.52 \text { to }+1.20)\end{array}$ & $\begin{array}{l}+0.12(0.28 \\
-0.78 \text { to }+1.00)\end{array}$ \\
\hline loss) (paired Student's t-test) & $P=0.000 \mathrm{I}$ & $P<0.000$ I \\
\hline $\begin{array}{l}\text { Mean gain in VA - difference } \\
\text { between groups (unpaired } \\
\text { Student's } t \text {-test) }\end{array}$ & $0.00, P=0.99$ & \\
\hline
\end{tabular}

Abbreviations: VA, visual acuity; logMAR, logarithm of the minimum angle of resolution; SD, standard deviation. 


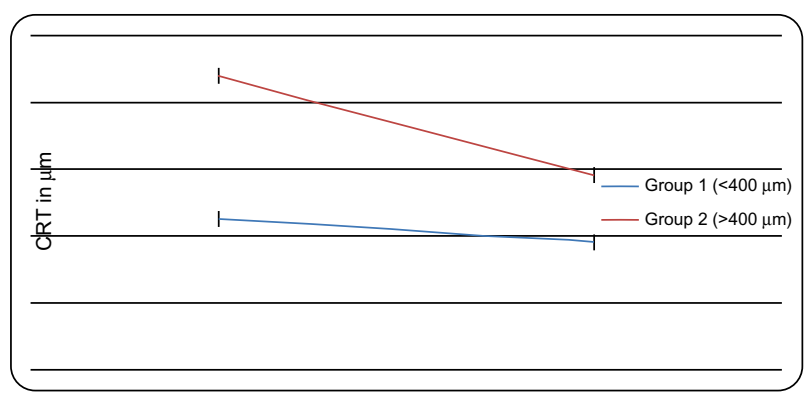

Figure I Comparison of initial and final CRT between groups I and 2 .

Notes: Standard error bars are included. Both groups showed a significant reduction in CRT between the baseline and month 12 , with a significantly greater reduction in group 2 compared to group 1 .

Abbreviation: CRT, central retinal thickness.

we could not demonstrate a significant difference in visual outcome between eyes with $>400 \mu \mathrm{m}$ or $<400 \mu \mathrm{m}$ of CRT due to DME but admittedly the entry criteria of this study differed from the ranibizumab clinical trials in that the mean baseline VA in our study was lower by about $0.2 \log$ MAR units (ten letters). Both groups had a modest improvement of about six letters (1.2 logMAR units).

To our knowledge, only one published study on bevacizumab in DME has performed subgroup analysis of visual outcome based on baseline CRT. ${ }^{19}$ In that study, Soheilian et $\mathrm{l}^{19}$ found that eyes with a baseline central macular thickness of $<250 \mu \mathrm{m}$ had significantly greater VA improvement than eyes with $>350 \mu \mathrm{m}$ but their follow-up period was only up to 36 weeks. It is also worthwhile to note that a reduction on foveal thickness has also been demonstrated in a study that used intravitreal pegaptanib. ${ }^{22}$

These results suggest that in terms of differential visual gain from treating DME with bevacizumab, the $350 \mu \mathrm{m}$ or $400 \mu \mathrm{m}$ baseline CRT cut-off is not currently supported

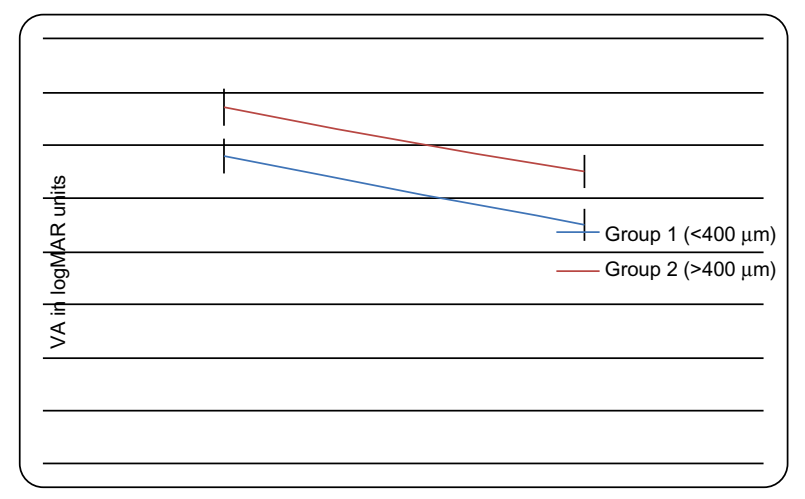

Figure 2 Comparison of initial and final VA between group I and group 2.

Notes: Standard error bars are included. Both groups gained by a mean of $0.12 \log$ MAR units between baseline and month 12. There was no statistical difference between groups $I$ and 2 in terms of vision gain.

Abbreviations: VA, visual acuity; logMAR, logarithm of the minimum angle of resolution. by the evidence from this study and from the published literature. This is in contrast to the published data on ranibizumab in DME. In the RESTORE study, for instance, eyes with a baseline CRT of $>400 \mu \mathrm{m}$ gained approximately six letters while the eyes with a baseline CRT of $<300 \mu \mathrm{m}$ gained only three letters at 12 months. ${ }^{10}$ In the Diabetic Retinopathy Clinical Research Network Protocol I study, eyes with a baseline CRT of $>400 \mu \mathrm{m}$ had a mean gain of eleven letters while eyes with $<400 \mu \mathrm{m}$ had a mean gain of seven letters at 2 years. ${ }^{13}$

A possible reason for the discrepancy in differential visual outcome between our study and those from large clinical trials could be due to the chronicity of DME. In our study, all patients had cystoid pattern on regrading of SD-OCT; this would indicate a population with more chronic DME. In the Diabetic Retinopathy Clinical Research Network Protocol I study, approximately $40 \%$ of all patients treated with ranibizumab did not have prior laser and, in the RESTORE study, the mean duration of DME prior to study entry was $<2$ years. ${ }^{10,13}$ Indeed, the chronicity of DME and the presence of cystoid spaces on SD-OCT were found by Shimura et al to be poor prognostic factors for visual improvement when treated with intravitreal bevacizumab. ${ }^{18}$

In this study, both groups had a small or modest gain in VA, despite achieving a considerable reduction in CRT. This may be explained by a poor correlation between the functional and the anatomical response, which has been described with bevacizumab and between triamcinolone and ranibizumab. ${ }^{13,21,23}$ The other explanation is the lack of correlation between CRT and VA, which is also well documented. ${ }^{24-26}$ The lack of correlation between macular thickness and VA suggests that an anatomical parameter, based on a finite threshold of macular thickness, is unlikely to be a good predictor of treatment response in terms of visual gain.

We also found that patients in group 2 received more injections than group 1. This would have the effect of making the treatment less cost-effective in patients with thicker CRT. The differential number of injections between subgroups in the RESTORE and protocol I studies was not taken into account when the cost-effectiveness of each subgroup was considered in the National Institute of Clinical Excellence appraisal of ranibizumab for DME. ${ }^{10,12,13}$ This may have led to an overestimation of the cost-effectiveness of treating the subgroup of patients with DME $>400 \mu \mathrm{m}$.

This retrospective case series has several limitations. Visual acuities were not measured with a refraction protocol at regular visits as in a prospective clinical trial. Criteria for 
retreatment could have been variable throughout the study. Therefore, the overall efficacy of bevacizumab therapy could not be judged from our visual outcome data. However, this is unlikely to have a great impact on the interpretation of the difference in treatment response between eyes with $>400 \mu \mathrm{m}$ or $<400 \mu \mathrm{m}$ of DME, as we performed SD-OCT regrading to ensure that both groups were balanced in terms of consistency of CRT measurements, presence of cystoid change, and confounding characteristics, such as epiretinal membrane. We were able to balance the two groups in terms of influencing factors, such as epiretinal membrane presence, sex, and age, but we did not have the ability to collect accurate data to demonstrate the balance of other influential factors such as the duration of diabetes and blood pressure. As the patients in this study were all at the severe end of the spectrum, we feel it is unlikely that this omission should affect our ability to answer the specific study question on the issue of a $400 \mu \mathrm{m}$ threshold. A final limitation was the absence of a ranibizumab control. Findings from bevacizumab-treated eyes may not translate to a ranibizumab scenario. The absence of an effect of a $400 \mu \mathrm{m}$ threshold seen in this study does not necessarily invalidate the $400 \mu \mathrm{m}$ threshold seen in the ranibizumab studies, such as the RESTORE trial.

The strength of this study lies in the consecutive nature of its fairly large series, which we feel reflects the unselected population that would present to any secondary care facility providing bevacizumab therapy for patients with DME. Therefore, our study results should be useful for practitioners who have to consider the use of bevacizumab in patients with CRT of either $>400 \mu \mathrm{m}$ or $<400 \mu \mathrm{m}$ without applying other exclusion criteria, such as DME duration, blood pressure, glycemic control, or SD-OCT pattern - as would be the case when translating from prospective randomized trials designed to evaluate efficacy. The issue of the unlicensed use of bevacizumab for the treatment of DME, when there is a licensed alternative (ranibizumab), is still a controversial one.

A recent review ${ }^{27}$ of published outcomes on the use of these two agents in various studies for this indication did not reveal any significant differences in clinical effectiveness and adverse events, and we agree with the authors of this review that a head-to-head study would provide a much-needed evidence base for those clinicians who have to continue using bevacizumab for treatment of DME.

\section{Conclusion}

In conclusion, we were unable to demonstrate a difference in functional outcome between patients who were grouped according to an anatomical threshold of $400 \mu \mathrm{m}$ of
CRT at baseline. Our results do not support a preferential selection of those with DME and CRT $>400 \mu \mathrm{m}$ at the baseline for intravitreal bevacizumab. On the contrary, we found that patients with thicker CRT required more injections rendering the treatment potentially less cost-effective. As there are no published studies to show equivalence between bevacizumab and ranibizumab for DME, it is not possible to say if we can translate these findings in the use of ranibizumab in DME.

\section{Author contributions}

BM conceived and designed the study with YY, participated in the data collection, and assisted with revising the manuscript. NC collected and analyzed the data, participated in the literature search, wrote the abstract, and suggested revisions to the manuscript. ATD collected the data, participated in the data analysis, and assisted with revising the manuscript. PLL made significant contributions to the data interpretation and revisions to the manuscript. PS and SE assisted in the study design and revisions to the manuscript. YY designed the study, interpreted the data, conducted the literature search, and wrote and revised the manuscript.

\section{Disclosure}

The authors report no conflicts of interest in this work.

\section{References}

1. Klein R, Klein BE, Moss SE, Davis MD, DeMets DL. The Wisconsin epidemiologic study of diabetic retinopathy. IV. Diabetic macular edema. Ophthalmology. 1984;91(12):1464-1474.

2. Funatsu H, Yamashita H, Noma H, Mimura T, Yamashita T, Hori S. Increased levels of vascular endothelial growth factor and interleukin- 6 in the aqueous humor of diabetics with macular edema. Am J Ophthalmol. 2002;133(1):70-77.

3. Jonas JB, Neumaier M. Vascular endothelial growth factor and basic fibroblast growth factor in exudative age-related macular degeneration and diffuse diabetic macular edema. Ophthalmic Res. 2007;39(3): 139-142.

4. Lee WJ, Kang MH, Seong M, Cho HY. Comparison of aqueous concentrations of angiogenic and inflammatory cytokines in diabetic macular oedema and macular oedema due to branch retinal vein occlusion. Br J Ophthalmol. 2012;96(11):1426-1430.

5. Cunningham ET Jr, Adamis AP, Altaweel M, et al; Macugen Diabetic Retinopathy Study Group. A phase II randomized double-masked trial of pegaptanib, an anti-vascular endothelial growth factor aptamer, for diabetic macular edema. Ophthalmology. 2005;112(10): 1747-1757.

6. Nguyen QD, Shah SM, Heier JS, et al; READ-2 Study Group. Primary End Point (Six Months) Results of the Ranibizumab for Edema of the mAcula in diabetes (READ-2) study. Ophthalmology. 2009;116(11): 2175-2181. e1.

7. Massin P, Bandello F, Garweg JG, Hansen LL, Harding SP, Larsen M, et al. Safety and efficacy of ranibizumab in diabetic macular edema (RESOLVE Study): a 12-month, randomized, controlled, double-masked, multicenter phase II study. Diabetes Care. 2010;33(11):2399-2405.

8. Michaelides M, Kaines A, Hamilton RD, et al. A prospective randomized trial of intravitreal bevacizumab or laser therapy in the management of diabetic macular edema (BOLT study) 12-month data: report 2. Ophthalmology. 2010;117(6):1078-1086. e2. 
9. Do DV, Schmidt-Erfurth U, Gonzalez VH, et al. The DA VINCI Study: phase 2 primary results of VEGF Trap-Eye in patients with diabetic macular edema. Ophthalmology. 2011;118(9):1819-1826.

10. Mitchell P, Bandello F, Schmidt-Erfurth U, et al; RESTORE study group. The RESTORE study: ranibizumab monotherapy or combined with laser versus laser monotherapy for diabetic macular edema. Ophthalmology. 2011;118(4):615-625.

11. Nguyen QD, Brown DM, Marcus DM, et al; RISE and RIDE Research Group. Ranibizumab for diabetic macular edema: results from 2 phase III randomized trials: RISE and RIDE. Ophthalmology. 2012;119(4):789-801.

12. National Institute for Health and Care Excellence. Macular oedema (diabetic) - ranibizumab: guidance. London: National Institute for Health and Care Excellence; 2013.

13. Diabetic Retinopathy Clinical Research Network, Elman MJ, Aiello LP, Beck RW, et al. Randomized trial evaluating ranibizumab plus prompt or deferred laser or triamcinolone plus prompt laser for diabetic macular edema. Ophthalmology. 2010;117(6):1064-1077. e35.

14. Kook PE, Maier M, Schuster T, Feucht N, Lohmann CP. Nine-month results of intravitreal bevacizumab versus triamcinolone for the treatment of diffuse diabetic macular oedema: a retrospective analysis. Acta Ophthalmol. 2011;89(8):769-773.

15. Roh MI, Kim JH, Kwon OW. Features of optical coherence tomography are predictive of visual outcomes after intravitreal bevacizumab injection for diabetic macular edema. Ophthalmologica. 2010;224(6): 374-380.

16. Wu PC, Lai CH, Chen CL, Kuo CN. Optical coherence tomographic patterns in diabetic macula edema can predict the effects of intravitreal bevacizumab injection as primary treatment. J Ocul Pharmacol Ther. 2012;28(1):59-64.

17. Framme C, Schweizer P, Imesch M, Wolf S, Wolf-Schnurrbusch U. Behavior of SD-OCT-detected hyperreflective foci in the retina of antiVEGF-treated patients with diabetic macular edema. Invest Ophthalmol Vis Sci. 2012;53(9):5814-5818.

18. Shimura M, Yasuda K, Yasuda M, Nakazawa T. Visual outcome after intravitreal bevacizumab depends on the optical coherence tomographic patterns of patients with diffuse diabetic macular edema. Retina. 2013;33(4):740-747.
19. Soheilian M, Ramezani A, Yaseri M, Mirdehghan SA, Obudi A, Bijanzadeh B. Initial macular thickness and response to treatment in diabetic macular edema. Retina. 2011;31(8):1564-1573.

20. Stefanini FR, Arevalo JF, Maia M. Bevacizumab for the management of diabetic macular edema. World J Diabetes. 2013;4(2):19-26.

21. Soheilian M, Garfami KH, Ramezani A, Yaseri M, Peyman GA. Twoyear results of a randomized trial of intravitreal bevacizumab alone or combined with triamcinolone versus laser in diabetic macular edema. Retina. 2012;32(2):314-321.

22. Rinaldi M, Chiosi F, dell'Omo R, et al. Intravitreal pegaptanib sodium (Macugen $^{\circledR}$ ) for treatment of diabetic macular oedema: a morphologic and functional study. Br J Clin Pharmacol. 2012;74(6):940-946.

23. Erol N, Gursoy H, Kimyon S, Topbas S, Colak E. Vision, retinal thickness, and foveal avascular zone size after intravitreal bevacizumab for diabetic macular edema. Adv Ther. 2012;29(4):359-369.

24. Otani T, Yamaguchi Y, Kishi S. Correlation between visual acuity and foveal microstructural changes in diabetic macular edema. Retina. 2010;30(5):774-780.

25. Forooghian F, Stetson PF, Meyer SA, et al. Relationship between photoreceptor outer segment length and visual acuity in diabetic macular edema. Retina. 2010;30(1):63-70.

26. Diabetic Retinopathy Clinical Research Network, Browning DJ, Glassman AR, Aiello LP, et al. Relationship between optical coherence tomography-measured central retinal thickness and visual acuity in diabetic macular edema. Ophthalmology. 2007;114(3): 525-536.

27. Ford JA, Elders A, Shyangdan D, Royle P, Waugh N. The relative clinical effectiveness of ranibizumab and bevacizumab in diabetic macular oedema: an indirect comparison in a systematic review. $B M J$. 2012;345:e5182.
Clinical Ophthalmology

\section{Publish your work in this journal}

Clinical Ophthalmology is an international, peer-reviewed journal covering all subspecialties within ophthalmology. Key topics include: Optometry; Visual science; Pharmacology and drug therapy in eye diseases; Basic Sciences; Primary and Secondary eye care; Patient Safety and Quality of Care Improvements. This journal is indexed on

\section{Dovepress}

PubMed Central and CAS, and is the official journal of The Society of Clinical Ophthalmology (SCO). The manuscript management system is completely online and includes a very quick and fair peer-review system, which is all easy to use. Visit http://www.dovepress.com/ testimonials.php to read real quotes from published authors. 